\title{
A Comparative Study of Techniques for Bone Age Assessment using Image Processing
}

\author{
Simerjeet Kaur \\ M.Tech (CE) Department UCOE, \\ Punjabi University Patiala \\ Punjab, India.
}

\begin{abstract}
Bone age assessment is an innovation which empowers us to determination the age bone with the help PC picture preparing and assessment of the computerized perceptions. In this review paper we have reviewed various methods for bone age assessment like active shape modeling random forest regression method, Greulich \& Pyle method, Tanner and Whitehouse method and RUS method with their advantages and disadvantages. All of the above methods provide effective assistance in processing phase of the bone age assessment.
\end{abstract}

\section{Keywords}

Bone age, Regression, Region of interest (ROIs), Fragment, Carpal Bone, Wrist Bone, Radiographic.

\section{INTRODUCTION}

In Forensic examination of Anatomy, the examination of skeleton remains is an on-going examination reliably. In this get some information about first rule trademark is, to assess the sex structure skeleton stays, second age estimation, third illnesses perceiving affirmation, fourth to comprehend reason behind death eventually the view of way of life (society), flourishing conditions and climatic changes reliably. [13][14] This study serves to look at the obvious changes in mankind's history. Amongst all the above, sex and age determinations are the most evident segments to be resolved. Regardless, all around when we get skeleton remains or half spoiled body because of trademark fiascoes (earth disasters, bomb influences and waves) it's hard to perceive the sexual presentation and age in these circumstances. [16] Along these lines, unmistakable investigators have picked sexual presentation and age utilizing different parts of the body uninhibitedly. Research in quantifiable is slammed into arranged extents of interest, one is for dead skeleton bones concentrate, half disintegrated human bodies (in wrongdoing examination) on the other hand direct for the live human (for skeleton change and remedial determination).

In creating individuals, the bone age is the most normally used premise of characteristic improvement and age. Bone age evaluations rely on upon considering the periods of improvement associated with skeletal headway through the appraisal of hand and wrist radiographies. [10] The level of skeletal advancement can, essentially, is determined in light of two qualities: The level of improvement in extents encountering solidifying, and the level of calcium hoarding in those regions. From most punctual stages, these two qualities take after a certain and specific case and course of occasions till adulthood. [11][12] Examines performed from the most punctual beginning stage of the twentieth century to the present have made use of left hand and wrist radiographies for examination purposes. There are certain ideal circumstances associated with using only the left hand as opposed to both hands. In particular, using one hand diminishes both the cost of strategies and the individual's presentation to radiation significantly. [17] The way that the left hand has lower chances of experiencing disasters and damage on account of the higher pervasiveness of right-handedness in numerous social requests, and the way that researchers playing out the basic studies on bone age examination favored using the left hand are the fundamental reasons why the left hand is used in bone age assessments. [18][19] In the International Agreement for the Unification of Anthropometric Measurements to be made on Living Subjects, masterminded in the midst of the Physical Anthropologists Congresses held in Monaco in 1906 and in Geneva in 1912, the decision was made that examinations on living persons should be performed on the left $50 \%$ of the body. In effectively coordinated studies, it was determined that differentiations between the two sides of the body were sufficiently minor, such that they would not fast bungles in the examination of skeletal change.

\section{RELATED WORK}

All Yuh et al. [1] proposed skeletal headway estimation or bone age examination of kids is an average procedure performed in pediatrics. The bone age outlined by authorities could move sometimes and the precision relies on upon masters' encounters. Recalling the choosing goal to give a predictable and more correct bone age appraisal, PC boned age evaluation frameworks are utilized. Once the epiphysis mix has begun the level of blend and association parts are poor down. They demonstrates a tally to evacuate and isolate the surface segments of the areas of fervor of phalanges close to radiographs at the later stage with a specific choosing goal to give a continuing and fit later stage bone age evaluation. They demonstrated estimation first uses wavelet change to get three motivations behind interest sub-clusters from every zone of interest. By then solitary quality breaking down is utilized on every sub-band to segregate the most awesome probability highlight, $\lambda$. At long last, fortify vector machine is related for depiction. Test results demonstrated that this figuring can give suitable and correct later stage bone age evaluation.

Wu et al. [2] represented a method is taken to be granted for different break mechanics considers have portrayed bone's solidness to break comparatively as crucial apprehension power section and vital strain centrality discharge rate measured at the onset of a break split. These concentrates, however critical, offer kept data into relief conduct of bone as, not in any way like set up fragile materials, bone is a miniaturized scale splitting strong that chooses its imperviousness to soften up the middle of the arrangement of split spread from small scale crack portions happening behind the driving break front. The goal of this study was to examine the common sense of an extension approach in recognizing age-related changes in bone strength. Start and augmentation tests drove on human cortical bone minimized outlines demonstrated specific degrees of age-related durability hardship in start and inducing. Wandered from start, inciting 
strength of cortical bone declined twice as quick and finished a close to zero quality at the tenth decade. These outcomes clearly plot that age-related diminishment in bone's break resistance might be more extraordinary than beginning now seen and that the impelling based measure might be more flimsy to age-related changes in bone.

Sonar et al. [3] discussed delineates a bone indicating exertion and virtual reality representation pointed towards depicting and suspecting human vertebral weight breaks with highlight on evaluating osteoporosis and creating. A 3D model of the trabecular bone structure is worked from a pile of muCT photographs of a human vertebra. Parameters for surveying the bone are resolved for this structure from the 3D show up and embraced by separating the outcomes and qualities figured from standard stereological systems. The subsequent structure is then created utilizing really based computational estimations. These structures are then envisioned in a virtual reality environment connecting with point by point examination of the provincial microstructures to survey the potential for weight break change.

Al-Tanni et al. [4] proposed another way to deal with oversee portraying bones of the hand-wrist pictures into pediatric times of change utilizing point course models (PDM). The technique includes two stages: the plan stage and the solicitation stage. Amidst get prepared, case of bones from every class are collected so that the tolerable shape mutilations for every class are learnt. A model tending to every class is made. These models are therefore used to total new example of the bones. Amidst depiction all shows are emerged from the information picture and the thing is doled out to the class whose model is the nearest encourage. Exploratory results acquired utilizing 120 photographs of the third distal and center phalanxes demonstrated the estimation of the methodology for social event these bones into their reasonable times of change.

Badiei et al. [5] represented delicacy of trabecular bone depends on upon the measure of bone and what's more on its arrangement. With a specific last target to survey delicacy of bone, portray changes in perspective of age, and screen impact of ailment or treatment, it is vital to demonstrate the physical properties of trabecular bone layout. An essential piece of bone building is the level of anisotropy (DA). Appraisals of DA might be taken from the care of tomography information by delineating presentation in pictures. Exhaustively utilized pictures of descriptors for surveying presentation in this are taken by setting the joint mean which are mean iterative length (MIL), star volume dispersal (SVD), star length scattering (SLD), and line part deviation (LFD). In this study, evaluations of DA enrolled by technique for each of these photograph descriptors are looked at on created pictures for different blends of trabecular thickness, bundle and number. Assessments of DA are likewise figured for true blue pictures tending to various times of creating. It is found that examinations of DA change through and through relying on the decision of picture descriptor. Specifically, the MIL has a tendency to consider little DA.

Stern et al. [6] studied determination of an individual's honest to goodness lion's offer age is winding up being progressively fundamental in honest to goodness rehearse. Created age estimation strategies depend on upon 2D X-columns, yet experience the insidious effects of issues by virtue of projective imaging and presentation to ionizing radiation, which, without reasonable therapeutic or criminal sign, is morally imperfect and lawfully limited in different nations. They have proposed a redone 3D strategy for the determination of genuine change from MR pictures considering the solidifying of the achieve bone. Age estimation is performed by a prompt lose the faith model of the epiphyseal hole volume over the known ground truth time of get prepared information. Results are in every practical sense undefined with the created Greulich/Pyle (GP) and Tanner/Whitehouse (TW) techniques, yet do prohibit risky radiation.

Shaikhina et al. [7] Artificial Neural Network (ANN) model has been presented to accomplice time of really osteoarthritic male and female case with key mechanical and key qualities of their trabecular bone. The whimsical interdependency between age, sexual presentation, compressive quality, porosity, morphology and level of interconnectivity was bankrupt down in multi-dimensional space utilizing a twolayer feed forward ANN. Masterminded by LevenbergMarquardt back spread estimation, the ANN satisfied lose the faith segment of $\mathrm{R}=96.3 \%$ between the predicted and target age when improved for the exploratory dataset. Results display a solid relationship of the 5-dimensional vector of physical properties of the bone with the age of the representations. The opposite issue of evaluating compressive quality as the key bone split danger was in like way researched. The results yield relationship amongst expected and target compressive quality with lose the faith segment of $\mathrm{R}=97.4 \%$. Inside the impediments of the information set, the ANNs give strong farsighted models to hard tissue building choice sponsorship.

\section{TYPES OF MODELS}

\subsection{Active shape models}

Model - based vision is ardently settled as a vivacious way to deal with oversee seeing and finding known unbendable articles inside seeing aggravation, obfuscate, and impediment. [8] It is more unsafe to apply model based frameworks to pictures of articles whose appearance can differentiate, however changed methodologies in context of the utilization of flexible configurations have been proposed. The issue with existing systems is that they surrender model specificity

Recalling the choosing target to suit variability, along these lines trading off power amidst picture perception. They have fought that a model ought to just be able to twist in courses run of the mill for the class of articles it addresses. They have delineated a system for building models by taking in the event of variability from a preparation set of exactly elucidated pictures. These models can be utilized for picture look as a part of an iterative refinement calculation undifferentiated from that utilized by Active Contour Models (Snakes). The key separation is that our Active Shape Models can basically mutilate to fit the information in courses obvious with the arranging set. The writers of this strategy display two or three functional circumstances where we have made such models and utilized them to find to some degree blocked articles in uproarious, messed pictures. The Active Shape Model guarantees model specificity that is the model is in pair with the state of the given picture of the article which is accomplished by including the shape imperatives. The Active Shape model is exceedingly vigorous to clamor and jumble. Then again, the production of the Active shape Model is unpredictable when contrasted with the Finite component model or the dynamic Contour model. This many-sided quality emerges from the need to stamp every one of the edges in the preparation set with the right elucidation. 


\subsection{Random forest regression voting based shape model}

An overall utilized system for discovering focuses on deformable articles in pictures is to make highlight reaction pictures for every point, and after that to fit a shape model to these reaction pictures. [9] They have shown that Random Forest fall away from the faith voting can be used to make incredible reaction pictures rapidly. As opposed to utilizing a generative or a discriminative model to review every pixel, a repressor was utilized to cast votes in favor of the ideal position of every point. They have displayed that this prompts energetic and precise shape model arranging when related in the Constrained Local Model structure. They have reviewed the framework in unnoticeable segment, and separation it and a degree of customarily utilized choices crosswise over application districts: the comment of the joints of the hands in radiographs and the affirmation of highlight focuses in facial pictures. They demonstrated that their system outflanks elective structures, accomplishing what we recognizes to be the most correct results yet passed on for hand joint comment and best in class execution for facial part point affirmation. The favorable position in this is utilizing a solitary vote for every tree gives great results, and is altogether quicker than option approaches. The hindrance is that the coarseness of the inspecting step must be conformed to adjust amongst rate and precision as required.

\subsection{Greulich and Pyle strategy}

In their strategy for each of these bones a detailed depiction of its formative stages is incorporated. [6][20] The depictions are increasingly a general rule to the advancement of every bone in the hand than a direction on the best way to ratea bone. Most foundations utilize a quicker adjusted rendition of the first, which is likewise possibly less precise. That form is portrayed underneath. Keeping in mind the end goal to decide the skeletal age utilizing the altered Greulich and Pyle strategy one uses the chart book that they have created. The upsides of utilizing this technique is this can decide the sex of the patient and separate manuals are made for male and females. Burden is that the exactness is traded off now and again in view of the intricacy.

\subsection{Tanner and Whitehouse technique}

The TW2 technique doesn't utilize a scale taking into account the age, rather it depends on an arrangement of bone's standard development for every age populace. [20] In points of interest, in the TW2 strategy twenty areas of interest (ROIs) situated in the principle bones are considered for the bone age assessment. Every ROI is partitioned in three sections: epiphysis, metaphysis and diaphysis expecially in youngsters, it is conceivable to distinguish these diverse solidification focuses in the phalanx closeness. The benefit of this strategy is that it utilizes the finger and the carpal bones. Burden is its dispersed methodology of various ROIs.

\subsection{RUS approach}

For The objective of this preprocessing is to standardize the picture dim scale so that the later division step will be more vigorous. [20] The system first fragments the whole hand (bones and tissue) from the foundation utilizing a thresholding operation. After this a model-based technique is utilized to discover the bones in the hand. This technique utilizes learning of the relative positions of the bones in the hand as for each other and to the shape of the hand. After the inexact position of a bone is discovered its form is given by a versatile shape taking after calculation. This strategy utilizes just the carpal bones. Preferred standpoint is that this technique is brisk yet the impediment is it doesn't utilize the finger bones.

\section{CONCLUSION}

All the techniques that we have discussed are playing their part in enhancing the bone age assessment technology. Mechanized strategies for assessment of hand and wrist radiographs are likewise being created which diminish entomb rater variability contrasted with manual techniques. Non radiation based strategies of picturing hand and wrist bones, for example, ultra Sonography for bone age estimation have been hypothesized however are not as exact as radiographic techniques. The active shape modeling and Random forest are used to for shape modeling. Active shape modeling is very robust which deals with the problem dynamically and the Random forest is a technique which is highly flexible depending upon the number of votes involved. Greulich and Pyle method aids us with the ability of sex determination and provides vital information. Tanner and Whitehouse method uses finger and carpal bones for bone age assessment whereas RUS method only uses carpal bone falling behind in accuracy but advancing in speed.

In the future work we will experiment with other regression techniques like Artificial Neural Networks based Regression to test the improvement of accuracy. Further, we will experiment with ICA instead of PCA in Active Shape Models to test the results.

\section{REFERENCES}

[1] Yuh, Y.S., Liu, C.C., Chang, J.D. and Yu, S.S., 2012, July. Later stage bone age assessment on hand radiographs. In Industrial Electronics and Applications (ICIEA), 2012 7th IEEE Conference on (pp. 1329-1332). IEEE.

[2] Wu, P.C. and Vashishth, D., 2002. Age-related changes in cortical bone toughness: initiation vs. propagation. In Engineering in Medicine and Biology, 2002. 24th Annual Conference and the Annual Fall Meeting of the Biomedical Engineering Society EMBS/BMES Conference, 2002. Proceedings of the Second Joint (Vol. 1, pp. 425-426). IEEE.

[3] Sonar, A.V., Carroll, J.J., Sabini, L. and Issen, K.A., 2008, May. Simulation of bone aging and virtual reality visualization of cancellous bone structure. In Electrical and Computer Engineering, 2008. CCECE 2008. Canadian Conference on (pp. 001317-001322). IEEE.

[4] Al-Taani, A.T., Ricketts, I.W. and Cairns, A.Y., 1996, October. Classification of hand bones for bone age assessment. In Electronics, Circuits, and Systems, 1996. ICECS'96. Proceedings of the Third IEEE International Conference on (Vol. 2, pp. 1088-1091). IEEE.

[5] Badiei, A., Bottema, M.J. and Fazzalari, N.L., 2005, December. Expected and observed changes to architectural parameters of trabecular bone with aging-A comparison of measurement techniques. In Digital Image Computing: Techniques and Applications, 2005. DICTA'05. Proceedings 2005(pp. 71-71). IEEE.

[6] Stern, D., Ebner, T., Bischof, H. and Urschler, M., 2014, April. Determination of legal majority age from 3D magnetic resonance images of the radius bone. In Biomedical Imaging (ISBI), 2014 IEEE 11th International Symposium on(pp. 1119-1122). IEEE. 
[7] Shaikhina, T., Khovanova, N.A. and Mallick, K.K., 2014, June. Artificial neural networks in hard tissue engineering: another look at age-dependence of trabecular bone properties in osteoarthritis. In Biomedical and Health Informatics (BHI), 2014 IEEEEMBS International Conference on (pp. 622-625). IEEE.

[8] Cootes, T.F., Taylor, C.J., Cooper, D.H. and Graham, J., 1995. Active shape models-their training and application. Computer vision and image understanding, 61(1), pp.3859.

[9] Lindner, C., Bromiley, P.A., Ionita, M.C. and Cootes, T.F., 2015. Robust and Accurate Shape Model Matching using Random Forest Regression-Voting. Pattern Analysis and Machine Intelligence, IEEE Transactions on 37(9), pp.1862.1874.

[10] S. A. Adeshina. Automatic Detennination of Skeletal Maturity using Statistical Models of appearance. PhD thesis, University of Manchester, 2010.

[11] S. A. Adeshina and T F. Cootes. Constructing part-based models for groupwise registration. In Proc. IEEE International Syposium on Biomedical Imaging, 2010.

[12] S. A. Adeshina, T F. Cootes, and J. Adams. Evaluating different structures for predicting skeletal maturity using statistical appearance models. In Proc. MIUA 2009, 2009.

[13] S. A. Adeshina, T F. Cootes, and J. Adams. Evaluating the use of carpal bones for determination of skeletal maturity for infants. In Proc. MIUA 2010, 2010.
[14] T F. Cootes, G. J. Edwards, and C. J. Taylor. Active appearance models. IEEE Transactions on Pattern Analysis and Machine Intelligence, 23(6):681-685, 2001.

[15] T F. Cootes, C. J. Taylor, D. Cooper, and J. Graham. Active shape models - their training and application. Computer Vision and Image Understanding, 61(1):38-59, Jan. 1995.

[16] D.Cristinacce and TF.Cootes. Automatic feature localization with constrained local models. Pattern Recognition, 41(10):3054-3067, 2008.

[17] D. Giordano, C. Spampinato, G. Scarciofalo, and R. Leonardi. An automatic system for skeletal bone age measurement by robust processing of carpal and epiphysial/metaphysial bones. Instrumentation and Measurement, IEEE Transactions on, 59(10):2539-2553, October 2010.

[18] C. Hsieh, T Liu, and T Jong. A fuzzy-based growth model with principle component analysis selection for carpal boneage assessment. Med Bioi Eng Compute, 48(6):579-588, 2010

[19] C. Lindner, S. Thiagarajah, J. M. Wilkinson, G. A. Wallis, and T F. Cootes. Accurate fully automatic femur segmentation in pelvic radiographs using regression voting. In MICCAI, pages 353-360, 2012.

[20] Spampinato, C., 1995. Skeletal Bone Age Assessment University of Catania, Viale Andrea Doria, 6, p.95125. 